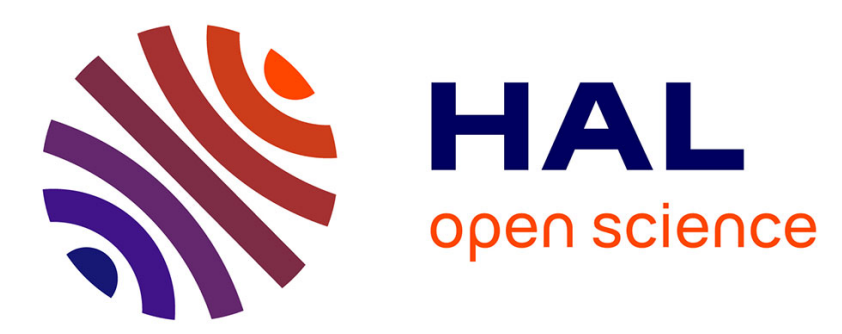

\title{
Acoustic radiation torque on a particle in a fluid: An angular spectrum based compact expression
}

Zhixiong Gong, Michael Baudoin

\section{To cite this version:}

Zhixiong Gong, Michael Baudoin. Acoustic radiation torque on a particle in a fluid: An angular spectrum based compact expression. Journal of the Acoustical Society of America, 2020, 148 (5), pp.3131-3140. 10.1121/10.0002491 . hal-03329995

\section{HAL Id: hal-03329995 \\ https://hal.science/hal-03329995}

Submitted on 31 Aug 2021

HAL is a multi-disciplinary open access archive for the deposit and dissemination of scientific research documents, whether they are published or not. The documents may come from teaching and research institutions in France or abroad, or from public or private research centers.
L'archive ouverte pluridisciplinaire HAL, est destinée au dépôt et à la diffusion de documents scientifiques de niveau recherche, publiés ou non, émanant des établissements d'enseignement et de recherche français ou étrangers, des laboratoires publics ou privés. 
Acoustic radiation torque on a particle in a fluid: An angular spectrum based compact expression

Zhixiong Gong, and Michael Baudoin

Citation: The Journal of the Acoustical Society of America 148, 3131 (2020); doi: 10.1121/10.0002491

View online: https://doi.org/10.1121/10.0002491

View Table of Contents: https://asa.scitation.org/toc/jas/148/5

Published by the Acoustical Society of America

Advance your science and career as a member of the 


\title{
Acoustic radiation torque on a particle in a fluid: An angular spectrum based compact expression
}

\author{
Zhixiong Gong ${ }^{\text {a) }}$ and Michael Baudoin ${ }^{\mathrm{b})}$ \\ Université de Lille, CNRS, Centrale Lille, Yncréa ISEN, Université Polytechnique Hauts-de-France, UMR 8520, IEMN, \\ F- 59000 Lille, France
}

\begin{abstract}
:
In this work, a set of compact analytical formulas is derived expressing the three-dimensional acoustic radiation torque (ART) exerted on a particle of arbitrary shape embedded in a fluid and insonified by an arbitrary acoustic field. This formulation enables direct computation of the ART from the angular spectrum based beam shape coefficients introduced by Sapozhnikov and Bailey [J. Acoust. Soc. Am. 133, 661-676 (2013)] and the partial wave coefficients. It is particularly well suited to determine the ART exerted on a particle when the acoustic field is known in a source plane. (C) 2020 Acoustical Society of America. https://doi.org/10.1121/10.0002491
\end{abstract}

(Received 6 April 2020; revised 19 October 2020; accepted 23 October 2020; published online 25 November 2020)

[Editor: Philip L. Marston]

Pages: 3131-3140

\section{INTRODUCTION}

The acoustic radiation torque (ART) exerted by an arbitrary acoustic field on a particle can, in general, be decomposed into three contributions: ${ }^{1}$ one resulting from the incident wave scattering by the particle, one induced by absorption of the acoustic field by the particle, ${ }^{2}$ and one resulting from the wave absorption in the viscous boundary layer surrounding the particle. ${ }^{3-5}$ All these contributions are nonlinear second order effects, and not first order as suggested by the misleading title in Ref. 3. In addition, the particle can also be set in rotation by the so-called Eckart streaming ${ }^{6}$ - a flow resulting from the thermo-viscous absorption of the wave in the bulk of the fluid-in particular, when the incident beam is carrying angular momentum. ${ }^{1,7,8}$ The ART can be calculated by transferring the integration of time-averaged stress tensor of angular momentum flux over the particle surface to a far-field spherical surface centered in the mass center of the particle, as first demonstrated by Maidanik. ${ }^{9,10}$

Based on this idea, Zhang and Marston ${ }^{11}$ derived a compact formula of the axial ART $\left(T_{z}\right)$ acting on an axisymmetric object centered on the axis of a cylindrical acoustical vortex beam beyond the paraxial approximation. The significance of energy dissipation in the vortex beam case was originally discussed in the paraxial limit. ${ }^{12}$ Zhang and Marston showed that, in this configuration, the scattering contribution vanishes and the ART is proportional to the absorbed power $\left(P_{a b s}\right)$ with a factor $M / \omega$, with $M$ the beam's topological charge and $\omega$ the angular frequency, i.e., $T_{z}=P_{a b s} M / \omega$. For a sphere in a Bessel vortex beam, the relation between dissipation and scattering was analyzed by Zhang and Marston with an explicit expression of $P_{a b s .}{ }^{10,13}$

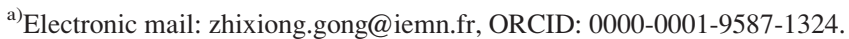

b) Also at: Institut Universitaire de France, 1 rue Descartes, 75005 Paris, France.
}

The theory applies for an elastic sphere in an inviscid fluid, and is also applicable for a sphere embedded in a weakly viscous fluid by modifying the scattering coefficients of the sphere. ${ }^{1,5,11,14}$ However, this theory is limited ${ }^{2}$ and cannot address the following situations: (i) non-axisymetric beams acting on a sphere (e.g., offset incidence of vortex beam on a sphere or oblique incidence on a spheroid and cylinder), (ii) non-axisymmetric objects with respect to the incident direction (e.g., broadside incidence on a spheroid), and (iii) multiple particles.

In 2012, Silva et al. ${ }^{15}$ used a spherical wave expansion of the incident and scattered beam-the so-called multipole expansion method (MEM) - to determine ART formulas applied on an arbitrary located sphere insonified by an arbitrary incident field in terms of the incident and scattered beam shape coefficients (BSC, the coefficients corresponding to the projection of the wavefields on the spherical wave basis). These ART formulas were recovered later on by Gong et $a$. $^{2}$ and extended to the case of arbitrary shape particles by using the T-matrix method. These theoretical developments were used to explore physical mechanisms at the origin of the three-dimensional (3D) torque reversal.

But one major difficulty with the MEM is to calculate the BSC for an arbitrary field and an arbitrary located sphere. Baresch et al. ${ }^{16}$ demonstrated that when the BSC are known for a specific sphere location, they can be determined for any configuration by translating and rotating the spherical basis with some numerical toolbox. Also, for specific wavefields it is possible to determine analytical expressions of the BSC for an arbitrary located particle. The most simple configuration is the plane wave, since in this case the BSCs do not depend on the sphere location owing to the wavefield symmetry. The case of cylindrical Bessel beam (CBB) was treated by Gong et al., ${ }^{17}$ who derived analytical expressions of the BSC for off-axis arbitrary incidence using Graf's addition theorem. This expression was recovered later on by 
Zhang et al. ${ }^{18}$ using a different method. Nevertheless, all these methods are difficult to set into practice when analytical expressions of the incident beam are not known. Recently, Zhao et al. ${ }^{19}$ evaluated three methods to determine the BSCs to compute the force for arbitrary (and in particular experimentally measured) acoustic fields: The first one relies on the orthogonality property of the spherical harmonics. From this property, the BSC can be obtained from a scalar product (integral over a spherical surface) of the wavefield and the spherical harmonics. This approach was investigated by Silva ${ }^{20}$ to solve the off-axis scattering problem. Nevertheless, this method (i) requires to know or measure the value of the acoustic field over a sphere surrounding the insonified particle and (ii) was shown to induce fluctuation when calculating the acoustic radiation force and might lead to similar issues when computing the ART. The second method relies on the knowledge/measurement of the acoustic field at random points in a spherical volume and on the resolution of the inverse problem by a sparse approach. Finally, the third method is based on the decomposition of the incident field into a sum of plane waves using the angular spectrum method (ASM) introduced by Sapozhnikov and Bailey ${ }^{21}$ for the calculation of the acoustic radiation force. The major advantages of this last method are that (i) only the knowledge of the field in one plane is required (though the source can be plane or curved) and (ii) that the ASMbased BSC can be computed from simple integration of the angular spectrum (the two-dimensional, 2D, spatial Fourier transform of the field in the reference plane) over a disk in the reciprocal space. Hence it is easy to set in practice, especially when using planar holographic transducers able to produce complex fields, such as acoustical vortices ${ }^{22-25}$ or when the acoustic field can be measured in a reference plane. ${ }^{19,26}$

In this paper, we derive a compact analytical formula expressing the torque applied by an arbitrary field on an arbitrary located particle of arbitrary shape and size as a function of the ASM-based BSCs. The formulas are validated through comparison with previous results obtained by Gong et $a l^{2}$ with the MEM for an off-axis viscoelastic sphere insonified by a CBB. Note that here (see Appendix $\mathrm{C})$, the BSC for CBB of off-axis arbitrary incidence is recovered with the ASM method. Finally, the potential of this approach is illustrated by calculating the torque applied on a $5 \mu \mathrm{m}$ particle insonified by a one-sided focused vortex beam produced by a plane active holographic transducer similar to the one used by Baudoin et al. ${ }^{25,27}$ to trap microparticles and cells.

\section{ANGULAR SPECTRUM BASED ART FORMULAS}

In this section, we give a brief overview of the main steps leading to the derivation of the angular spectrum based ART formulas (with the same notations as in Ref. 2). The ART exerted by an acoustic field on a particle can be calculated by transferring the integration of the time-averaged $(\langle\cdot\rangle)$ stress tensor of the angular momentum flux over the particle surface to a far-field spherical surface $S_{0}{ }^{2,9,10,15}$ Based on the divergence theorem, the integral expression of the ART is

$$
\mathbf{T}=-\rho_{0} \iint_{S_{0}}\langle L\rangle \mathbf{r} \times d \mathbf{S}-\rho_{0} \iint_{S_{0}}\langle(\mathbf{r} \times \mathbf{u}) \mathbf{u}\rangle d \mathbf{S},
$$

where $\left.\langle L\rangle=\langle 1 / 2 \mathbf{u} \cdot \mathbf{u})-p^{2} /\left(2 \rho_{0} c_{0}^{2}\right)\right\rangle$ is the time-averaged acoustic Lagrangian, $\mathbf{r}$ is the field point, $p$ is the total acoustic pressure field (incident + scattered), $\mathbf{u}$ is the total acoustic velocity vector, $\rho_{0}$ is the fluid density at rest, $c_{0}$ is the fluid sound speed, and $d \mathbf{S}=\mathbf{n} \cdot r^{2} \sin \theta d \theta d \varphi$ is the differential surface in the far field with $\mathbf{n}$ the outward unit normal vector. If $S_{0}$ is a sphere whose center coincide with the referential center, then $\mathbf{r} \times \mathbf{n}=r \mathbf{n} \times \mathbf{n}=0$ and the first term in Eq. (1) vanishes. Then, the incident (index "i") and scattered (index "s") acoustic velocity $\mathbf{u}$ and pressure $p$ fields can be described in terms of acoustical potentials $\Phi$ as

$$
\mathbf{u}_{i, s}=\nabla \Phi_{i, s} \text { and } p_{i, s}=i \omega \rho_{0} \Phi_{i, s},
$$

with $i$ is the imaginary unit and $\omega$ the angular frequency. Hence, the ART expression in Eq. (1) can be written in terms of the incident $\left(\Phi_{i}\right)$ and scattered $\left(\Phi_{s}\right)$ velocity potentials as

$$
\mathbf{T}=\frac{\rho_{0}}{2} \operatorname{Im}\left\{\iint_{S_{0}}\left(\frac{\partial \Phi_{i}^{*}}{\partial r} \mathbf{L} \Phi_{s}+\frac{\partial \Phi_{s}^{*}}{\partial r} \mathbf{L} \Phi_{i}+\frac{\partial \Phi_{s}^{*}}{\partial r} \mathbf{L} \Phi_{s}\right) d S\right\},
$$

where "Im" designates the imaginary part, the star superscript the complex conjugate and $\mathbf{L}=-i(\mathbf{r} \times \nabla)$ is the angular momentum operator, with its components in the three directions and their recursion relations with normalized spherical harmonics given in detail in Appendix A.

Now, assuming that the incident pressure field is known in a plane defined as $z=0,\left.p_{i}\right|_{z=0}=p_{i}(x, y, 0)$, the angular spectrum $S\left(k_{x}, k_{y}\right)$ of the acoustic field (that is nothing but the 2D spatial Fourier transform of the complex temporal harmonic amplitude of the field in this plane) reads

$$
S\left(k_{x}, k_{y}\right)=\int_{-\infty}^{+\infty} \int_{-\infty}^{+\infty} p_{i}(x, y, 0) e^{-i k_{x} x-i k_{y} y} d x d y
$$

with $x$ and $y$ the cartesian coordinates in the plane, $k_{x}$ and $k_{y}$ the wavenumber components in $x$ and $y$ directions. Then, the field at any point can be calculated by propagating each plane wave composing the source plane up to the target point $(x, y, z)$,

$$
\begin{aligned}
p_{i}(x, y, z)= & \frac{1}{4 \pi^{2}} \iint_{k_{x}^{2}+k_{y}^{2} \leq k^{2}} S\left(k_{x}, k_{y}\right) \\
& \times e^{i k_{x} x+i k_{y} y+i \sqrt{k^{2}-k_{x}^{2}-k_{y}^{2} z}} d k_{x} d k_{y},
\end{aligned}
$$

where $k=\omega / c_{0}$ is the wave number in fluid. In this way, the acoustic field is decomposed into an infinite sum of plane waves and the angular spectrum $S\left(k_{x}, k_{y}\right)$ characterizes 
the relative magnitude of each plane wave. The next step is to solve the scattering problem. For this purpose, this plane wave decomposition must be turned into a spherical wave decomposition, more suitable to solve the scattering problem (see Sapozhnikov and Bailey ${ }^{21}$ ),

$$
p_{i}=\frac{1}{\pi} \sum_{n=0}^{\infty} \sum_{m=-n}^{n} i^{n} H_{n m} j_{n}(k r) Y_{n m}(\theta, \varphi),
$$

where $Y_{n m}(\theta, \varphi)$ are the spherical harmonics and the $H_{n m}$ represent the respective weight of each spherical wave and hence are nothing but ASM-based BSCs,

$$
H_{n m}=\iint_{k_{x}^{2}+k_{y}^{2} \leq k^{2}} S\left(k_{x}, k_{y}\right) Y_{n m}^{*}\left(\theta_{k}, \varphi_{k}\right) d k_{x} d k_{y},
$$

with $\cos \theta_{k}=\left[1-\left(k_{x}^{2}+k_{y}^{2}\right) / k^{2}\right]^{1 / 2}$ and $\varphi_{k}=\arctan \left(k_{y} / k_{x}\right)$. This expression results from the known decomposition of a plane wave into a sum of spherical waves. Analytical solutions of the scattering problem for spheres embedded in a fluid are known in many cases including rigid, ${ }^{28}$ elastic, ${ }^{29}$ or viscoelastic particles. ${ }^{1,2}$ For non-spherical particles, the scattering problem can be handled with the so-called T-matrix method. ${ }^{30-35}$ Assuming prior knowledge of the scattering coefficients, the scattered field can be written under the form

$$
p_{s}=\frac{1}{\pi} \sum_{n=0}^{\infty} \sum_{m=-n}^{n} i^{n} H_{n m} A_{n m} h_{n}^{(1)}(k r) Y_{n m}(\theta, \varphi),
$$

with $A_{n m}$ the partial wave coefficients which only depend on the index $n$ for a spherical shape, having $A_{n}=\left(s_{n}-1\right) / 2$ with $s_{n}$ the scattering coefficients, and depend on both $n$ and $m$ for non-spherical shapes. ${ }^{2}$

Now the incident and scattered pressure fields are given in terms of the BSC $H_{n m}$ based on the ASM. ${ }^{21}$ The incident $\left(\Phi_{i}\right)$ and scattered $\left(\Phi_{s}\right)$ velocity potentials can be easily obtained by using the second equation of Eq. (2), which can then be substituted into Eq. (3). Since the integral is performed on the far field surface $S_{0}$, the asymptotic expressions of Bessel functions can be used [Eq. (A1) in Appendix A], which combined to the recursion relation of Bessel functions [Eqs. (A2) in Appendix A] leads to the following expression of the ART expression in terms of $H_{n m}$ and $Y_{n}^{m}$ :

$$
\begin{aligned}
\mathbf{T}= & -\frac{1}{2 \pi^{2} \rho_{0} k^{3} c_{0}^{2}} \operatorname{Re}\left\{\sum_{n=0}^{\infty} \sum_{m=-n}^{n} \sum_{n=0}^{\infty} \sum_{m^{\prime}=-n^{\prime}}^{n^{\prime}}\left(1+A_{n}^{m^{*}}\right) A_{n^{\prime}}^{m^{\prime}}\right. \\
& \left.\times H_{n m}^{*} H_{n^{\prime} m^{\prime}} \iint_{S_{0}}\left(Y_{n}^{m^{*}} \mathbf{L} Y_{n^{\prime}}^{m^{\prime}}\right) \sin \theta d \theta d \varphi\right\} .
\end{aligned}
$$

The final compact expression of the 3D ART in terms of the $H_{n m}$ coefficients can be derived by using the recursion and orthogonality relations of the normalized spherical harmonics (see details in Appendix B),

$$
T_{x}=-\frac{1}{4 \pi^{2} \rho_{0} k^{3} c_{0}^{2}} \operatorname{Re}\left\{\sum_{n=0}^{\infty} \sum_{m=-n+1}^{n} b_{n}^{m} C_{n}^{m} H_{n m}^{*} H_{n, m-1}\right\},
$$

$$
\begin{aligned}
& T_{y}=-\frac{1}{4 \pi^{2} \rho_{0} k^{3} c_{0}^{2}} \operatorname{Im}\left\{\sum_{n=0}^{\infty} \sum_{m=-n+1}^{n} b_{n}^{m} C_{n}^{m} H_{n m}^{*} H_{n, m-1}\right\}, \\
& T_{z}=-\frac{1}{2 \pi^{2} \rho_{0} k^{3} c_{0}^{2}} \operatorname{Re}\left\{\sum_{n=0}^{\infty} \sum_{m=-n}^{n} m D_{n}^{m} H_{n m}^{*} H_{n m}\right\},
\end{aligned}
$$

with $b_{n}^{m}=\sqrt{(n-m+1)(n+m)}, C_{n}^{m}=A_{n}^{m-1}+2 A_{n}^{m-1} A_{n}^{m *}$ $+A_{n}^{m *}, D_{n}^{m}=A_{n}^{m}+A_{n}^{m} A_{n}^{m *}$. Note that the prior ART formulas by Silva et al. ${ }^{15}$ were not written in a compact form since they do not express the scattered BSC in terms of the product of the incident BSC and $A_{n}$ for a spherical shape and have index issues. ${ }^{36} \mathrm{~A}$ thorough comparison between the present formula and the one obtained by Silva et al. ${ }^{15}$ is provided in Ref. 37.

\section{VALIDATION OF THE ANGULAR SPECTRUM BASED ART FORMULAS FOR AN OFF-AXIS VISCO- ELASTIC SPHERE INSONIFIED BY A BESSEL BEAM}

To validate the ART expressions obtained with the ASM in the previous section, the torque exerted on an offaxis viscoelastic sphere insonified in an inviscid fluid by a cylindrical Bessel vortex is calculated with Eqs. (10)-(12) and compared with the results obtained with the $\mathrm{MEM}^{17}$ by Gong et $a l .{ }^{2}$ Note that for a sphere in a vortex beam, there are two forms of rotations when the sphere is off the beam axis: (i) the orbital rotation of the sphere around the beam axis induced by the azimuthal force, and (ii) the spin rotation of the sphere around its own mass center by the torque. For a non-absorbing sphere located on the axis of the vortex, there is no axial torque following the theory proposed by Zhang and Marston ${ }^{11}\left(T_{z}=P_{a b s} M / \omega\right)$ since $P_{a b s}=0$. In this configuration, analytical expression of the $H_{n m}$ coefficients is given by (see detailed analytical derivation with ASM in Appendix C 2)

$$
\begin{aligned}
H_{n m}= & 4 \pi^{2} \omega \rho_{0} \Phi_{0} \xi_{n m} i^{M-m+1} P_{n}^{m}(\cos \beta) \\
& \times J_{m-M}\left(k_{\perp} R_{0}\right) e^{-i k_{z} z_{0}} e^{i(M-m) \varphi_{0}}
\end{aligned}
$$

where $\xi_{n m}=[(2 n+1)(n-m) !]^{1 / 2}[4 \pi(n+m) !]^{-1 / 2}, \quad M$ is the topological charge of the Bessel beam, $\beta$ is the cone angle, $k_{\perp}=k \sin \beta, R_{0}=\sqrt{x_{0}^{2}+y_{0}^{2}}$, and $x_{0}, y_{0}$, and $z_{0}$ are the offset along the $x, y$, and $z$ directions, respectively. The axial component of the wave number is $k_{z}=k \cos \beta$, and the original azimuthal angle is $\varphi_{0}=\tan ^{-1}\left(y_{0} / x_{0}\right)$. Note also that Eq. (13) obtained here with ASM is equivalent to previous theoretical expression obtained by Gong et al. ${ }^{17}$ and Zhang. ${ }^{18}$ By inserting Eq. (13) into Eq. (12), the expression of axial ART is verified equivalent to Eq. (15a) of Ref. 14. In the simulations represented on Fig. 1, the topological charge of the $\mathrm{CBB}$ is $M=1$ with $\beta=60^{\circ}$, the incident frequency is $1 \mathrm{MHz}$ with pressure amplitude $1 \mathrm{MPa}$, and the particle radius is $a=180 \mu \mathrm{m}$. For brevity, the acoustic parameters of the viscoelastic polyethylene (PE) sphere immersed in water are same as those used in Ref. 2, as given 
(a)

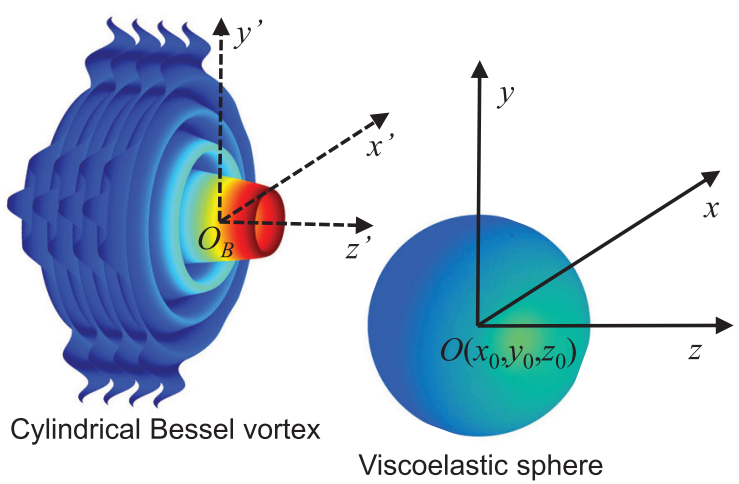

(b)

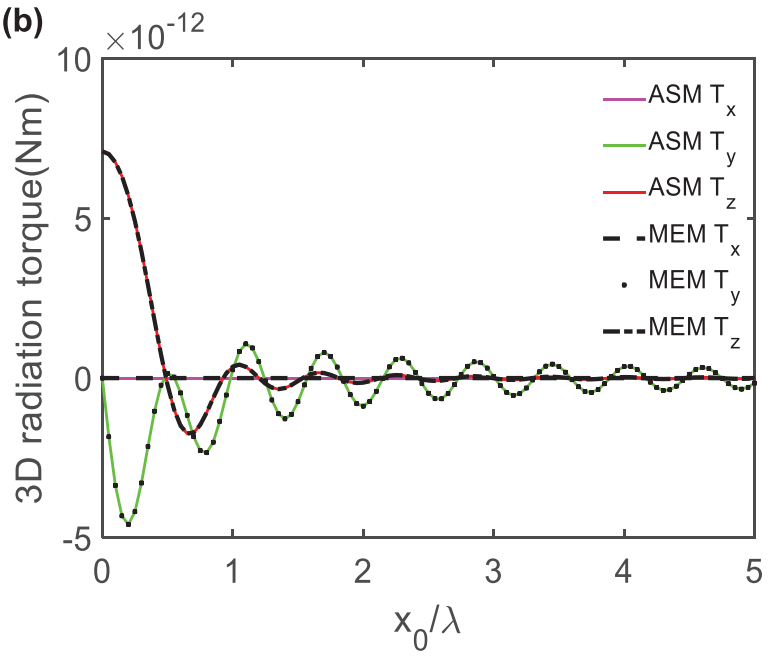

FIG. 1. (Color online) The three projections of the ART $\left(T_{x}, T_{y}, T_{z}\right)$ exerted on an off-axis viscoelastic sphere by a cylindrical Bessel vortex on a viscoelastic PE solid sphere are calculated by the present ASM [Eqs. (10)-(12)] and compared to results obtained with the MEM (Gong et al.) (Ref. 2). (a) Scheme of the simulated configuration. The topological order of the CBB is $M=1$ and the cone angle $\beta=60^{\circ}$ (see Ref. 36 for more details about acoustical vortices). The particle is moved away from the beam center along $x$ direction of a distance $x_{0} \in[0,5 \lambda]$, with $\lambda$ the wavelength in the fluid. There is no offset along the $y$ direction $\left(y_{0}=0\right)$ and since cylindrical Bessel are invariant along $z$, the position along this axis does not matter. (b) Figure comparing the values of the three projections of the ART obtained with ASM and MEM, as a function of the particle dimensionless offset $x_{0} / \lambda$.

in Table I. The particle is moved off the beam axis [see the schematic in Fig. 1(a)] along only $x$ direction with $x_{0} \in[0,5 \lambda], y_{0}=0$ and $z_{0}=0$ where $\lambda=1.5 \mathrm{~mm}$ is the wavelength in water. As observed in Fig. 1(b), the calculated 3D ART with the ASM [see Eqs. (10)-(12)] agree exactly with those by the MEM. ${ }^{2,37}$ No computational error is expected between the two methods since the two theoretical expressions can be shown to be equivalent. ${ }^{37}$ Note that the partial wave (or scattering) coefficients $A_{n m}$ for a viscoelastic sphere of both results are obtained based on the Kelvin-Voigt linear viscoelastic model ${ }^{38}$ with the explicit expressions given in the Appendix of Ref. 2. Further confirmation of these formula is under way by comparing directly the analytical expressions of the two formulas, ${ }^{37}$ i.e., the relation between the BSC can turn the ART formulas in Eqs. (10)-(12) into those by Silva et al. ${ }^{15}$ if index issues are improved. Note that since the particle is moved off axis along the $x$ direction, the lateral ART $T_{x}$ always vanishes

TABLE I. Acoustic parameters of particle materials and water. The absorption values for the PE were derived from ultrasonic measurements made by Hartmann and Jarsynski (Ref. 39) with the longitudinal and shear absorption per wavelength 0.4 and $1.2 \mathrm{~dB}$, respectively, while for the PS by Takagi et al. (Ref. 40) with the longitudinal and shear absorption coefficients 23 and $108 \mathrm{~Np} / \mathrm{m}$ at frequency $f=5 \mathrm{MHz}$. Note that $1 \mathrm{~Np}=8.6859 \mathrm{~dB}$. The normalized longitudinal $\left(\gamma_{p}\right)$ and shear $\left(\gamma_{s}\right)$ absorption coefficients are normalized by the corresponding wave number $\left(k_{p, s}\right)$ which are calculated by the longitudinal $\left(c_{p}\right)$ and shear $\left(c_{s}\right)$ velocities with $\omega=k_{p, s} c_{p, s}$.

\begin{tabular}{lccccc}
\hline \hline Material & Density $\left(\mathrm{kg} / \mathrm{m}^{3}\right)$ & $c_{p}(\mathrm{~m} / \mathrm{s})$ & $c_{s}(\mathrm{~m} / \mathrm{s})$ & $\gamma_{p}$ & $\gamma_{s}$ \\
\hline PE & 957 & 2430 & 950 & 0.0074 & 0.022 \\
PS & 1050 & 2350 & 1100 & 0.0017 & 0.0038 \\
Water & 1000 & 1500 & $\ldots$ & $\cdots$ & $\cdots$ \\
\hline \hline
\end{tabular}

because of the symmetry. For the normal incidence $\left(x_{0}=0\right.$ and $y_{0}=0$ ), only the axial ART due to acoustic absorption exists. ${ }^{1,11}$

\section{STUDY OF THE ART EXERTED BY A ONE-SIDED SPHERICAL VORTEX ON A VISCOELASTIC SPHERE}

To illustrate the potential of the present approach, we now apply our method to a case which cannot be treated analytically: the torque exerted on a $5 \mu \mathrm{m}$ viscoelastic polystyrene (PS) particle insonified by a $40 \mathrm{MHz}$ one-sided spherical vortex synthesized in an inviscid fluid by plane active spiraling transducers [Fig. 2(a)] similar to the one used by Baudoin et al. ${ }^{25,27}$ to trap microparticles and cells. The transducers are made of two active spiraling electrodes of inverse polarity whose equations are given in Ref. 25, exciting a piezoelectric wafer. In these simulations, the maximum radius of the transducer is $R_{t}=2.1 \mathrm{~mm}$ and the transducer is designed to obtain a focal plane located at $z=1 \mathrm{~mm}$ from the source plane, leading to a large aperture angle $65^{\circ}$ to obtain axial and hence $3 \mathrm{D}$ trapping capabilities. The acoustic pressure in the source plane $p_{i}(x, y, 0)$ is approximated as having the same geometrical distribution as the active spiral electrodes as shown in Fig. 2(a). That is to say, for the red electrode, the pressure has amplitude $1 \mathrm{MPa}$ and phase 0 , written as $p_{i}(x, y, 0)=1 \mathrm{MPa}$, while for the blue one, the pressure has amplitude $1 \mathrm{MPa}$ and phase $\pi$, written as $p_{i}(x, y, 0)=-1 \mathrm{MPa}$. The rest of the domain has a pressure equal to $p_{i}(x, y, 0)=0$. In the following computations, we take the physical domain as $3 R_{t} \times 3 R_{t}$ in $(x, y)$ plane and the fine mesh number 3000 with the physical space interval $R_{t} / 1000$ to assure the convergence. Based on the $2 \mathrm{D}$ fast Fourier transform, the angular spectrum is 

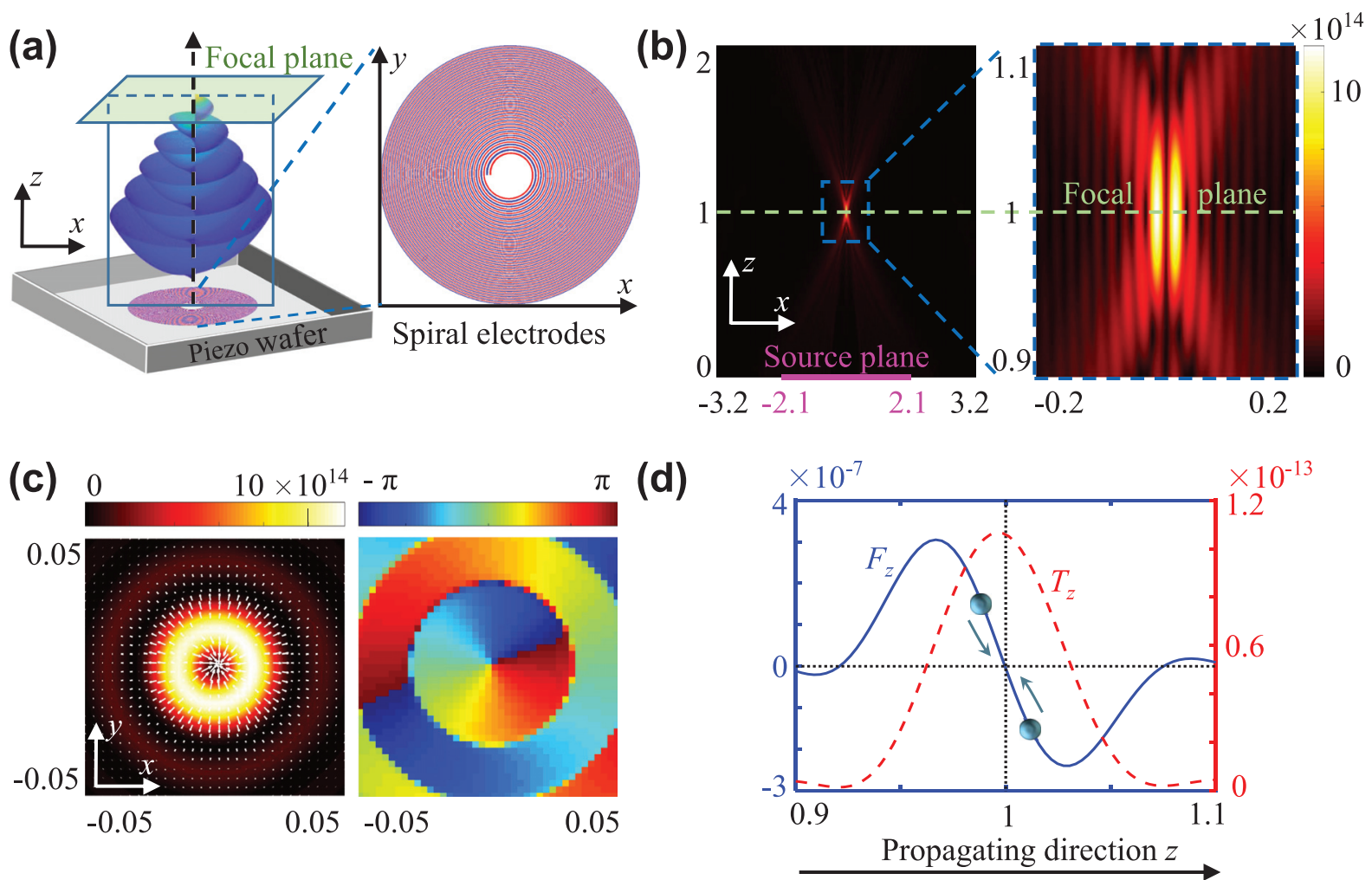

FIG. 2. (Color online) (a) Schematic of a one-sided focused vortex synthesized (left) with a set of two spiraling electrodes of inverse polarity (right, red and blue). The focal plane is designed at $z=1 \mathrm{~mm}$ with the source plane at $z=0$. The aperture of the spiral electrodes is $R_{t}=2.1 \mathrm{~mm}$. (b) Acoustic pressure magnitude square in the $(x, z)$ plane (left) with a zoom near the focal point (right). The lengths along $x$ and $z$ are not scaled. (c) Acoustic pressure magnitude square and phase in the lateral focus plane $(x, y)$ at $z=1 \mathrm{~mm}$. The arrows on the first panel give the lateral force for a viscoelastic PS particle with $a=5 \mu \mathrm{m}$ in radius in the focal plane, showing a slightly out-centered lateral trap. (d) The axial radiation force ( $F_{z}$ in Newton) and torque ( $T_{z}$ in $\mathrm{N}$ m) versus $z$ along the lateral trap axis. The particle is trapped in 3D [see the first panel of (c) for lateral trap and the blue line for axial trap] and spins around its mass center located on the beam axis. The lengths are in the unit of $\mathrm{mm}$ in (b)-(d).

directly computed with the wave number interval $2 \pi /\left(3 R_{t}\right)$ and the range $2000 \pi / R_{t} \times 2000 \pi / R_{t}$ in $\left(k_{x}, k_{y}\right)$ plane. The torque is computed with the formula provided in this paper while the force is computed using formulas by Sapozhnikov and Bailey. ${ }^{21}$ The field synthesized by these transducers computed with ASM is illustrated in Fig. 2(b) for the $(x, z)$ plane and Fig. 2(c) for the $(x, y)$ plane. The lateral force applied in the focal plane on the particle is shown as arrows on Fig. 2(c) (left). This figure shows that the particle trap position is a bit out-centered, which can be simply explained by the spiral finiteness. The axial force and torque is then calculated on the lateral trapping axis and represented in Fig. 2(d). This figure shows that spiraling transducers exhibit 3D trapping capabilities providing that the aperture is sufficient (here we chose an aperture of $65^{\circ}$ ). Note that the 3D trapping of $190-390 \mu \mathrm{m}$ PS particles in the $\mathrm{MHz}$ range with a focused vortex was demonstrated first theoretically and then experimentally with a complex array of transducers in Refs. 41 and 42. It also shows that the axial torque is maximum for a value slightly below the focal plane.

\section{CONCLUSIONS AND DISCUSSIONS}

In summary, some compact angular spectrum based 3D ART formulas are derived for a single particle immersed in an ideal fluid with no limitation to the particle size, particle shape, and beam shape structure. The arbitrary acoustic field is taken as the superposition of plane waves, hence can be expanded based on the $\mathrm{ASM},{ }^{21}$ which is quite practical for finite-aperture real sources. The ART on non-spherical shapes (e.g., spheroid and finite cylinder) can be calculated once the partial wave coefficients $A_{n}^{m}$ are obtained with proper methods, for example, the T-matrix method. ${ }^{2,30-35}$ The present theory is still practicable for an absorbing sphere in a viscous fluid if the absorption processes in the particle and viscous layer is accounted in the expression of scattering coefficients. ${ }^{1,5,11,14}$ In addition, the formulas can be used for multiple objects ${ }^{1,43,44}$ that are all located inside the chosen far-field spherical shape so that the divergence theorem still holds for the derivation. The ART of particle in experimental sources can be evaluated by the measured acoustic field in the transverse plane as similar to the simulation of acoustic radiation force. ${ }^{19}$ By combining with the $3 \mathrm{D}$ acoustic radiation forces, ${ }^{21}$ we can predict the dynamic motions of particles in real acoustic field with six degrees of freedom, i.e., three for translocations and three for spinning motions. It is noteworthy that for a particle located off the axis of a vortex beam, both $3 \mathrm{D}$ radiation forces and torques are applied on the particle so that the particle could rotate around the beam axis (by the azimuthal component of radiation force) and its own center of mass (by the radiation 
torque). Since a particle in a vortex beam could be ejected out of the trap, ${ }^{45}$ this work can be used for theoretical guidance on parameters selection of acoustic sources for experimental designs, which can slow down the spinning motions by decreasing the ART, and meanwhile, keep the trapping by the acoustic radiation force. In addition, this work has the potential to dynamically control the rotation and translation of particles in manipulation devices in and beyond the longwavelength regime. ${ }^{46,47}$

\section{ACKNOWLEDGMENTS}

We acknowledge the support of the programs ERC Generator and Prematuration funded by ISITE Université Lille Nord-Europe.

\section{APPENDIX A: LADDER OPERATORS}

The far-field asymptotic expressions of the spherical Bessel function and Hankel function of the first kind are, respectively,

$$
\begin{aligned}
& j_{n}(k r) \simeq i^{-(n+1)} e^{i k r} / 2 k r+i^{n+1} e^{-i k r} / 2 k r \\
& h_{n}^{(1)}(k r) \simeq i^{-(n+1)} e^{i k r} / k r
\end{aligned}
$$

and the recursion relation of the spherical Bessel function is

$$
j_{n}^{\prime}(k r)=(n / k r) j_{n}(k r)-j_{n+1}(k r)
$$

where the symbol ' means the derivative with respect to $k r$. The ladder operators $L_{ \pm}$has the relationship with the lateral components of the angular momentum operator $L_{x, y}$ : $L_{ \pm}=L_{x} \pm i L_{y}{ }^{48}$ The recursion relations of ladder operators $L_{ \pm}$(or axial component of angular momentum operator $L_{z}$ ) and normalized spherical harmonics are ${ }^{49}$

$$
\begin{aligned}
& L_{+} Y_{n}^{m}=b_{n}^{-m} Y_{n}^{m+1}, \\
& L_{-} Y_{n}^{m}=b_{n}^{m} Y_{n}^{m-1}, \\
& L_{z} Y_{n}^{m}=m Y_{n}^{m} .
\end{aligned}
$$

Finally, the orthogonality relation of the normalized spherical harmonics is 48

$$
\int_{0}^{2 \pi} d \varphi \int_{0}^{\pi} \sin \theta d \theta Y_{n}^{m *}(\theta, \varphi) Y_{n^{\prime}}^{m^{\prime}}(\theta, \varphi)=\delta_{n n^{\prime}} \delta_{m m^{\prime}} .
$$

\section{APPENDIX B: DERIVATION OF 3D ART IN TERMS OF $H_{N M}$}

\section{Detailed derivation of $T_{x}$}

Based on the ART formulas of Eq. (9), the expression of $x$-component of ART is

$$
\begin{aligned}
T_{x}= & -\frac{1}{2 \pi^{2} \rho_{0} k^{3} c_{0}^{2}} \operatorname{Re}\left\{\sum_{n=0}^{\infty} \sum_{m=-n}^{n} \sum_{n^{\prime}=0}^{\infty} \sum_{m^{\prime}=-n^{\prime}}^{n^{\prime}}\left(1+A_{n}^{m^{*}}\right)\right. \\
& \left.\times A_{n^{\prime}}^{m^{\prime}} H_{n m}^{*} H_{n^{\prime} m^{\prime}} \iint_{S_{0}} Y_{n}^{m^{*}} L_{x} Y_{n^{\prime}}^{m^{\prime}} \sin \theta d \theta d \varphi\right\} .
\end{aligned}
$$

Substitute Eqs. (A3a) and (A3b) into Eq. (B1) with the relation $L_{x}=\left(L_{+}+L_{-}\right) / 2$,

$$
\begin{aligned}
T_{x}= & -\frac{1}{4 \pi^{2} \rho_{0} k^{3} c_{0}^{2}} \operatorname{Re}\left\{\sum_{n=0}^{\infty} \sum_{m=-n}^{n} \sum_{n^{\prime}=0}^{\infty} \sum_{m^{\prime}=-n^{\prime}}^{n^{\prime}}\left(1+A_{n}^{m^{*}}\right)\right. \\
& \left.\times A_{n^{\prime}}^{m^{\prime}} H_{n m}^{*} H_{n^{\prime} m^{\prime}} \iint_{S_{0}} Y_{n}^{m^{*}}\left(L_{+}+L_{-}\right) Y_{n^{\prime}}^{m^{\prime}} \sin \theta d \theta d \varphi\right\} \\
= & -\frac{1}{4 \pi^{2} \rho_{0} k^{3} c_{0}^{2}} \operatorname{Re}\left\{\sum_{n=0}^{\infty} \sum_{m=-n}^{n} \sum_{n^{\prime}=0}^{\infty} \sum_{m^{\prime}=-n^{\prime}}^{n^{\prime}-1}\left(1+A_{n}^{m^{*}}\right)\right. \\
& \times A_{n^{\prime}}^{m^{\prime}} H_{n m}^{*} H_{n^{\prime} m^{\prime}} \iint_{S_{0}} Y_{n}^{m^{*}} b_{n^{\prime}}^{-m^{\prime}} Y_{n^{\prime}}^{m^{\prime}+1} \sin \theta d \theta d \varphi \\
& +\sum_{n=0}^{\infty} \sum_{m=-n}^{n} \sum_{n^{\prime}=0}^{\infty} \sum_{m^{\prime}=-n^{\prime}+1}^{n^{\prime}}\left(1+A_{n}^{m^{*}}\right) \\
& \left.\times A_{n^{\prime}}^{m^{\prime}} H_{n m}^{*} H_{n^{\prime} m^{\prime}} \iint_{S_{0}} Y_{n}^{m^{*}} b_{n^{\prime}}^{m^{\prime}} Y_{n^{\prime}}^{m^{\prime}-1} \sin \theta d \theta d \varphi\right\} .
\end{aligned}
$$

Note that the regimes of $\left(n^{\prime}, m^{\prime}\right)$ in the summation symbol is based on the definition of the normalized spherical harmonics (i.e., $Y_{n^{\prime}}^{m^{\prime}}$ and $Y_{n^{\prime}}^{m^{\prime} \pm 1}$ ), which are the intersection part and listed in Table II.

Using the orthogonality relationship in Eq. (A4), the expression of $T_{x}$ is

$$
\begin{aligned}
T_{x}= & -\frac{1}{4 \pi^{2} \rho_{0} k^{3} c_{0}^{2}} \operatorname{Re}\left\{\sum_{n=0}^{\infty} \sum_{m=-n}^{n} \sum_{n^{\prime}=0}^{\infty} \sum_{m^{\prime}=-n^{\prime}}^{n^{\prime}-1}\left(1+A_{n}^{m^{*}}\right)\right. \\
& \times A_{n^{\prime}}^{m^{\prime}} H_{n m}^{*} H_{n^{\prime} m^{\prime}} b_{n^{\prime}}^{-m^{\prime}} \delta_{n n^{\prime}} \delta_{m, m^{\prime}+1} \\
& +\sum_{n=0}^{\infty} \sum_{m=-n}^{n} \sum_{n^{\prime}=0}^{\infty} \sum_{m^{\prime}=-n^{\prime}+1}^{n^{\prime}}\left(1+A_{n}^{m *}\right) \\
& \left.\times A_{n^{\prime}}^{m^{\prime}} H_{n m}^{*} H_{n^{\prime} m^{\prime}} b_{n^{\prime}}^{m^{\prime}} \delta_{n n^{\prime}} \delta_{m, m^{\prime}-1}\right\} \\
= & -\frac{1}{4 \pi^{2} \rho_{0} k^{3} c_{0}^{2}} \operatorname{Re}\left\{\sum_{n=0}^{\infty} \sum_{m=-n+1}^{n}\left(1+A_{n}^{m^{*}}\right)\right. \\
& \times A_{n}^{m-1} H_{n m}^{*} H_{n, m-1} b_{n}^{-m+1}+\sum_{n=0}^{\infty} \sum_{m=-n}^{n-1}\left(1+A_{n}^{m *}\right) \\
& \left.\times A_{n}^{m+1} H_{n m}^{*} H_{n, m+1} b_{n}^{m+1}\right\} .
\end{aligned}
$$

Here, we use a re-index for the second part of Eq. (B3) by using a variable substitution $q=m+1 \in[-n+1, n]$, and note that $b_{n}^{-m+1}=b_{n}^{m}$,

TABLE II. Regime of $\left(n^{\prime}, m^{\prime}\right)$ in normalized spherical harmonics for derivation of $F_{x}$ and $F_{y}$. Note that based on the definition in Eq. (6), we have $n^{\prime} \in[0, \infty]$ and $m^{\prime} \in\left[-n^{\prime}, n^{\prime}\right]$.

\begin{tabular}{cccc}
\hline \hline & $n^{\prime}$ & $m^{\prime}$ & Intersection \\
\hline$Y_{n^{\prime}+1}^{m^{\prime}+1}$ & $n^{\prime} \in[0, \infty]$ & $m^{\prime} \in\left[-n^{\prime}-1, n^{\prime}-1\right]$ & $n^{\prime} \in[0, \infty], m^{\prime} \in\left[-n^{\prime}, n^{\prime}-1\right]$ \\
$Y_{n^{\prime}}^{m^{\prime}-1}$ & $n^{\prime} \in[0, \infty]$ & $m^{\prime} \in\left[-n^{\prime}+1, n^{\prime}+1\right]$ & $n^{\prime} \in[0, \infty], m^{\prime} \in\left[-n^{\prime}+1, n^{\prime}\right]$ \\
\hline \hline
\end{tabular}




$$
\begin{aligned}
T_{x} & =-\frac{1}{4 \pi^{2} \rho_{0} k^{3} c_{0}^{2}} \operatorname{Re}\left\{\sum_{n=0}^{\infty} \sum_{m=-n+1}^{n}\left(1+A_{n}^{m *}\right) A_{n}^{m-1} H_{n m}^{*} H_{n, m-1} b_{n}^{m}+\sum_{n=0}^{\infty} \sum_{q=-n+1}^{n}\left(1+A_{n}^{q-1 *}\right) A_{n}^{q} H_{n, q-1}^{*} H_{n, q} b_{n}^{q}\right\} \\
& =-\frac{1}{4 \pi^{2} \rho_{0} k^{3} c_{0}^{2}} \operatorname{Re}\left\{\sum_{n=0}^{\infty} \sum_{m=-n+1}^{n}\left(1+A_{n}^{m *}\right) A_{n}^{m-1} H_{n m}^{*} H_{n, m-1} b_{n}^{m}+\sum_{n=0}^{\infty} \sum_{m=-n+1}^{n}\left(1+A_{n}^{m-1 *}\right) A_{n}^{m} H_{n, m-1}^{*} H_{n, m} b_{n}^{m}\right\} \\
& =-\frac{1}{4 \pi^{2} \rho_{0} k^{3} c_{0}^{2}} \operatorname{Re}\left\{\sum_{n=0}^{\infty} \sum_{m=-n+1}^{n} b_{n}^{m}\left(A_{n}^{m-1}+2 A_{n}^{m-1} A_{n}^{m^{*}}+A_{n}^{m *}\right) H_{n m}^{*} H_{n, m-1}\right\},
\end{aligned}
$$

which is Eq. (10) in Sec. II. Note that $\operatorname{Re}\{X\}=\operatorname{Re}\left\{X^{*}\right\}$ with $X$ an arbitrary complex number.

\section{Derivation of $T_{y}$}

The expression of $y$-component of ART is

$$
T_{y}=-\frac{1}{2 \pi^{2} \rho_{0} k^{3} c_{0}^{2}} \operatorname{Re}\left\{\sum_{n=0}^{\infty} \sum_{m=-n}^{n} \sum_{n^{\prime}=0}^{\infty} \sum_{m^{\prime}=-n^{\prime}}^{n^{\prime}}\left(1+A_{n}^{m *}\right) A_{n^{\prime}}^{m^{\prime}} H_{n m}^{*} H_{n^{\prime} m^{\prime}} \iint_{S_{0}} Y_{n}^{m^{*}} L_{y} Y_{n^{\prime}}^{m^{\prime}} \sin \theta d \theta d \varphi\right\} .
$$

As similar as the derivation for $T_{x}$, the final expression of $T_{y}$ in terms of $H_{n m}$ can be obtained by using Eqs. (A3a) and (A3b) into Eq. (B5) and $L_{y}=\left(L_{+}-L_{-}\right) / 2 i$ instead of $L_{x}$, as given in Eq. (11) and omitted here for brevity.

\section{Detailed derivation of $T_{z}$}

The expression of $z$-component of ART is

$$
T_{z}=-\frac{1}{2 \pi^{2} \rho_{0} k^{3} c_{0}^{2}} \operatorname{Re}\left\{\sum_{n=0}^{\infty} \sum_{m=-n}^{n} \sum_{n^{\prime}=0}^{\infty} \sum_{m^{\prime}=-n^{\prime}}^{n^{\prime}}\left(1+A_{n}^{m^{*}}\right) A_{n^{\prime}}^{m^{\prime}} H_{n m}^{*} H_{n^{\prime} m^{\prime}} \iint_{S_{0}} Y_{n}^{m^{*}} L_{z} Y_{n^{\prime}}^{m^{\prime}} \sin \theta d \theta d \varphi\right\} .
$$

Inserting Eq. (A3c) into Eq. (B6) and using the orthogonality relation in Eq. (A4), the final expression of $T_{z}$ in terms of $H_{n m}$ can be derived as

$$
\begin{aligned}
T_{z} & =-\frac{1}{2 \pi^{2} \rho_{0} k^{3} c_{0}^{2}} \operatorname{Re}\left\{\sum_{n=0}^{\infty} \sum_{m=-n}^{n} \sum_{n^{\prime}=0}^{\infty} \sum_{m^{\prime}=-n^{\prime}}^{n^{\prime}}\left(1+A_{n}^{m^{*}}\right) A_{n^{\prime}}^{m^{\prime}} H_{n m^{*}}^{*} H_{n^{\prime} m^{\prime}} \iint_{S_{0}} Y_{n}^{m^{*}} m^{\prime} Y_{n^{\prime}}^{m^{\prime}} \sin \theta d \theta d \varphi\right\} \\
& =-\frac{1}{2 \pi^{2} \rho_{0} k^{3} c_{0}^{2}} \operatorname{Re}\left\{\sum_{n=0}^{\infty} \sum_{m=-n}^{n} \sum_{n^{\prime}=0}^{\infty} \sum_{m^{\prime}=-n^{\prime}}^{n^{\prime}}\left(1+A_{n}^{m^{*}}\right) A_{n^{\prime}}^{m^{\prime}} H_{n m}^{*} H_{n^{\prime} m^{\prime}} m^{\prime} \delta_{n n^{\prime}} \delta_{m m^{\prime}}\right\} \\
& =-\frac{1}{2 \pi^{2} \rho_{0} k^{3} c_{0}^{2}} \operatorname{Re}\left\{\sum_{n=0}^{\infty} \sum_{m=-n}^{n} m\left(1+A_{n}^{m^{*}}\right) A_{n}^{m} H_{n m}^{*} H_{n m}\right\},
\end{aligned}
$$

which is Eq. (12) in Sec. II.

\section{APPENDIX C: THEORETICAL DERIVATION OF ON- AND OFF-AXIS CBB BASED ON ASM}

\section{1. $H_{n m}$ for an on-axis CBB}

The $H_{n m}$ coefficients for an on axis particle insonified by a CBB were calculated analytically with the ASM by Sapozhnikov and Bailey. ${ }^{21}$ However, they only provided the result in the paper. Since the calculation is not straightforward, we give here the main elements of the demonstration before extending it to the case of off-axis CBB. The expression of a CBB is given by

$$
p_{i}(x, y, z)=p_{0} e^{i k_{\|} z} J_{M}\left(k_{\perp} R\right) e^{i M \varphi},
$$

with $k_{\|}=k \cos \beta, k_{\perp}=k \sin \beta, k=\omega / c_{o}$ the wavenumber, $\beta$ the cone angle, $R=\sqrt{x^{2}+y^{2}}$ the radius in cylindrical coordinates, and $\varphi=\arctan (y / x)$ in $\mathbb{R}^{3}$ space and $\left(O, \mathbf{e}_{\mathbf{z}}\right)$ is the central axis of the Bessel beam. In Cartesian coordinates, the angular spectrum in a plane $(x, y)$ of arbitrary altitude $z$ orthogonal to the Bessel beam central axis can be written as

$$
S\left(k_{x}, k_{y}\right)=\int_{x=-\infty}^{+\infty} \int_{y=-\infty}^{+\infty} d x d y p_{i}(x, y, z) e^{-i \mathbf{k} \cdot \mathbf{r}}
$$

with $\mathbf{k}=k_{x} \mathbf{e}_{\mathbf{x}}+k_{y} \mathbf{e}_{\mathbf{y}}+k_{z} \mathbf{e}_{\mathbf{z}}, k_{z}=\sqrt{k^{2}-k_{x}^{2}-k_{y}^{2}}$, and the expression of the position vector is $\mathbf{r}=x \mathbf{e}_{\mathbf{x}}+y \mathbf{e}_{\mathbf{y}}+z \mathbf{e}_{\mathbf{z}}$. This expression gives Eq. (4) when $z=0$ (a condition which can always be fulfilled with a simple change of frame of reference).

In cylindrical coordinates, the angular spectrum can be recast as 


$$
S\left(k_{R}, \varphi_{k}\right)=\int_{\varphi=0}^{2 \pi} \int_{R=0}^{+\infty} R d R d \varphi p_{i}(R, \varphi, z) e^{-i \mathbf{k} \cdot \mathbf{r}},
$$

with $\quad \mathbf{k}=k_{R} \mathbf{e}_{\mathbf{R}}\left(\varphi_{k}\right)+k_{z} \mathbf{e}_{\mathbf{z}}, \quad k_{R}=\sqrt{k_{x}^{2}+k_{y}^{2}}, \quad \varphi_{k}$ $=\arctan \left(k_{y} / k_{x}\right)$, and the position vector $\mathbf{r}=R \mathbf{e}_{\mathbf{r}}(\varphi)+z \mathbf{e}_{\mathbf{z}}$.

Inserting the expression of the CBB [Eq. (C1)] in Eq. (C3) and considering the angular spectrum in the plane $z=0$ gives

$$
\begin{aligned}
S\left(k_{R}, \varphi_{k}\right)= & p_{0} \int_{\varphi=0}^{2 \pi} \int_{R=0}^{+\infty} R d R d \varphi J_{M}\left(k_{\perp} R\right) \\
& \times e^{i\left[M \varphi-k_{R} R \cos \left(\varphi_{k}-\varphi\right)\right]} .
\end{aligned}
$$

From the integral definition of a cylindrical Bessel function, $J_{\alpha}(x)=(1 / 2 \pi) \int_{-\pi}^{\pi} e^{i(\alpha \tau-x \sin \tau)} d \tau$ and the variable substitution $\pi / 2-\phi=\varphi_{k}-\varphi($ so that $d \phi=d \varphi$ and $\cos (\pi / 2-\phi)$ $=\sin \phi)$, the integral of the exponential function over $d \varphi$ becomes

$$
\int_{0}^{2 \pi} d \varphi e^{i\left[M \varphi-k_{R} R \cos \left(\varphi_{k}-\varphi\right)\right]}=2 \pi i^{-M} J_{M}\left(k_{R} R\right) e^{i M \varphi_{k}} .
$$

Inserting Eq. (C5) into Eq. (C4) and combining the results with the orthogonality relation of cylindrical Bessel functions $\int_{0}^{\infty} x J_{\alpha}(u x) J_{\alpha}(v x) d x=\delta(u-v) / u$ gives

$$
\begin{aligned}
S\left(k_{R}, \varphi_{k}\right) & =p_{0} \int_{0}^{+\infty} R d R J_{M}\left(k_{\perp} R\right)\left[2 \pi i^{-M} J_{M}\left(k_{R} R\right) e^{i M \varphi_{k}}\right] \\
& =2 \pi p_{0} i^{-M} \frac{\delta\left(k_{\perp}-k_{R}\right)}{k_{\perp}} e^{i M \varphi_{k}},
\end{aligned}
$$

which is Eq. (62) in Ref. 21. Note that the dirac function is $\neq 0$ only when $k_{R}=k_{\perp}$. Hence, following the definition of $H_{n m}$ in terms of $S\left(k_{x}, k_{y}\right)$ [Eq. (7)], the integral over the disk domain $\left(k_{x}^{2}+k_{y}^{2} \leq k^{2}\right.$, i.e., $\left.k_{R}^{2}<k^{2}\right)$ degenerates into the integral over a circle corresponding to $k_{R}=k_{\perp}$, which in cylindrical coordinates becomes

$$
\begin{aligned}
H_{n m} & =\int_{0}^{2 \pi} k_{\perp} d \varphi_{k}\left[2 \pi p_{0} i^{-M} \frac{1}{k_{\perp}} e^{i M \varphi_{k}}\right] Y_{n}^{m *}\left(\theta_{k}, \varphi_{k}\right) \\
& =2 \pi^{3 / 2} p_{0} i^{-m} \sqrt{\frac{(2 n+1)(n-m) !}{(n+m) !}} P_{n}^{m}(\cos \beta) \delta_{M m},
\end{aligned}
$$

with $Y_{n}^{m *}=\sqrt{[(2 n+1) / 4 \pi][(n-m) ! /(n+m) !]} P_{n}^{m}\left(\cos \theta_{k}\right) e^{-i m \varphi_{k}}$ the conjugates of the spherical harmonics and $\int_{0}^{2 \pi} e^{i(M-m) \varphi_{k}} d \varphi_{k}=2 \pi \delta_{M m}$. This formula agrees with the expression given in Ref. 21. Note that in Fourier space, we have $\quad d k_{x} d k_{y}=R d k_{R} d \varphi_{k}, \quad$ and $\quad \cos \theta_{k}=\sqrt{1-k_{R}^{2} / k^{2}}$ $=\sqrt{1-k_{\perp}^{2} / k^{2}}=\cos \beta$.

\section{2. $H_{n m}$ for an off-axis CBB}

Now let us consider two frames of reference, $\mathcal{R}^{\prime}=\left(O^{\prime}\right.$, $\left.\left(\mathbf{e}_{\mathbf{x}}, \mathbf{e}_{\mathbf{y}}, \mathbf{e}_{\mathbf{z}}\right)\right)$ and $\mathcal{R}=\left(O,\left(\mathbf{e}_{\mathbf{x}}, \mathbf{e}_{\mathbf{y}}, \mathbf{e}_{\mathbf{z}}\right)\right)$, with the point $O^{\prime}$ located on the central axis of the Bessel beam and the point $O$ corresponding to the "off-axis" center of the particle, see Fig. 3. Hence the position vectors in the two reference frames are such

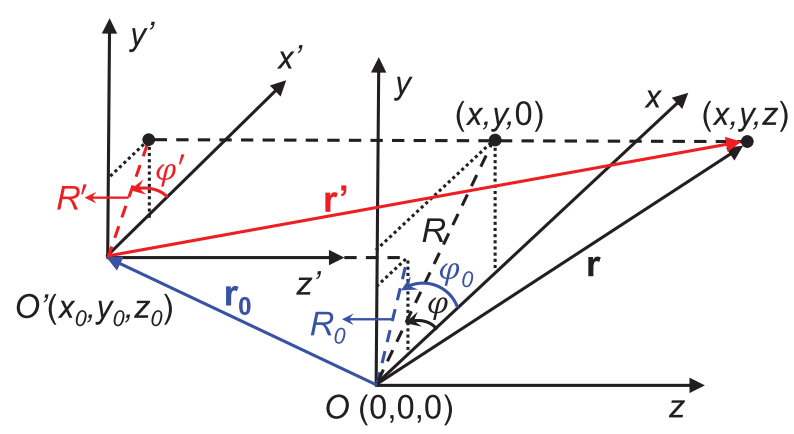

FIG. 3. (Color online) The global $O(x, y, z)$ with its origin $(0,0,0)$ at the particle center and local coordinates $O^{\prime}\left(x^{\prime}, y^{\prime}, z^{\prime}\right)$ with its origin $\left(x_{0}, y_{0}, z_{0}\right)$ at the beam center.

that: $\quad \mathbf{r}=\mathbf{r}_{0}+\mathbf{r}^{\prime}, \quad$ with $\quad \mathbf{r}_{0}=\mathbf{O O}^{\prime}=x_{0} \mathbf{e}_{\mathbf{x}}+y_{0} \mathbf{e}_{\mathbf{y}}+z_{0} \mathbf{e}_{\mathbf{z}}$ $=R_{o} \mathbf{e}_{\mathbf{r}}\left(\varphi_{0}\right)+z_{0} \mathbf{e}_{\mathbf{z}}$. The incident field in $\mathcal{R}^{\prime}$ is given by

$$
p_{i}\left(x^{\prime}, y^{\prime}, z^{\prime}\right)=p_{0} e^{i k_{\|} z^{\prime}} J_{M}\left(k_{\perp} R^{\prime}\right) e^{i M \varphi^{\prime}}
$$

where $\left(R^{\prime}, \varphi^{\prime}, z^{\prime}\right)$ are the cylindrical coordinates of $\mathbf{r}^{\prime}$ in $\mathcal{R}^{\prime}$.

The angular spectrum $S^{\prime}$ and $S$ in the planes $\left(O^{\prime},\left(\mathbf{x}^{\prime}, \mathbf{y}^{\prime}\right)\right)$ and $\left(O,\left(\mathbf{e}_{\mathbf{x}}, \mathbf{e}_{\mathbf{y}}\right)\right)$ are respectively given by

$$
\begin{aligned}
& S^{\prime}\left(k_{x}, k_{y}\right)=\int_{x=-\infty}^{+\infty} \int_{y=-\infty}^{+\infty} d x^{\prime} d y^{\prime} p_{i}\left(x^{\prime}, y^{\prime}, z^{\prime}\right) e^{-i \mathbf{k} \cdot \mathbf{r}^{\prime}}, \quad(\mathrm{C} 9) \\
& S\left(k_{x}, k_{y}\right)=\int_{x=-\infty}^{+\infty} \int_{y=-\infty}^{+\infty} d x d y p_{i}\left(x-x_{0}, y-y_{0}, z-z_{0}\right) e^{-i \mathbf{k} \cdot \mathbf{r}} .
\end{aligned}
$$

Since (i) the integral over $x$ and $y$ are infinite (and thus not modified by a translation), (ii) $p_{i}\left(x, y, z-z_{0}\right)=e^{-i k_{\|} z_{0}}$ $p_{i}(x, y, z)$, (iii) k.r $=\mathbf{k} \cdot \mathbf{r}^{\prime}+\mathbf{k} \cdot \mathbf{r}_{0}=\mathbf{k} \cdot \mathbf{r}^{\prime}+k_{R} \cos \left(\varphi_{k}-\varphi_{0}\right)$, we see directly that,

$$
S\left(k_{x}, k_{y}\right)=S^{\prime}\left(k_{x}, k_{y}\right) e^{-i k_{\|} z_{0}-i k_{R} \cos \left(\varphi_{k}-\varphi_{0}\right)} .
$$

The on-axis value of the angular spectrum $S^{\prime}$ was calculated in the previous section [Eq. (C6)], leading to

$$
\begin{aligned}
S\left(k_{R}, \varphi_{k}\right)= & 2 \pi i^{-M} p_{0} e^{-i k_{\|} z_{0}} e^{i\left[M \varphi_{k}-k_{R} R_{0} \cos \left(\varphi_{k}-\varphi_{0}\right)\right]} \\
& \times \frac{\delta\left(k_{\perp}-k_{R}\right)}{k_{\perp}} .
\end{aligned}
$$

We can now proceed very similarly to the on-axis case to compute the $H_{n m}$ coefficients and turn the integral over a disk into an integral over the circle $k_{R}=k_{\perp}$ :

$$
\begin{aligned}
H_{n m}= & \int_{0}^{2 \pi} k_{\perp} d \varphi_{k}\left[2 \pi i^{-M} p_{0} e^{-i k_{\|} z_{0}} e^{i\left[M \varphi_{k}-k_{\perp} R_{0} \cos \left(\varphi_{k}-\varphi_{0}\right)\right]}\right. \\
& \left.\times \frac{1}{k_{\perp}}\right] Y_{n}^{m *}\left(\theta_{k}, \varphi_{k}\right) \\
= & 2 \pi i^{-M} p_{0} e^{-i k_{\|} z_{0}} \sqrt{\frac{(2 n+1)}{4 \pi} \frac{(n-m) !}{(n+m) !}} P_{n}^{m}\left(\cos \theta_{k}\right) \\
& \times \int_{\varphi_{k}=0}^{2 \pi} d \varphi_{k}\left[e^{\left.i\left[(M-m) \varphi_{k}-k_{\perp} R_{0} \cos \left(\varphi_{k}-\varphi_{0}\right)\right]\right] .}\right.
\end{aligned}
$$


Using a variable substitution $\pi / 2-\phi=\varphi_{0}-\varphi_{k}$ (so that $\left.d \varphi_{k}=d \phi\right)$ to evidence the integral definition of the Bessel function [similar to what was done to obtain Eq. (C5)], Eq. (C12) turns out to be

$$
\begin{aligned}
H_{n m}= & 2 \pi^{3 / 2} i^{-m} p_{0} e^{-i k_{\|} z_{0}} \sqrt{\frac{(2 n+1)(n-m) !}{(n+m) !}} \\
& \times P_{n}^{m}(\cos \beta) e^{i(M-m) \varphi_{0}} J_{m-M}\left(k_{\perp} R_{0}\right),
\end{aligned}
$$

with $\cos \theta_{k}=\cos \beta$, and $J_{M-m}\left(k_{\perp} R_{0}\right)=(-1)^{m-M} J_{m-M}$ $\left(k_{\perp} R_{0}\right)$ for an integer $(M-m) \in \mathbb{Z}$. When the particle is located on the axis of a CBB [i.e., $\left(x_{0}, y_{0}, z_{0}\right)=(0,0,0)$ so that $\left.\left(R_{0}, \varphi_{0}, z_{0}\right)=(0,0,0)\right]$, Eq. (C13) degenerates into Eq. (C7). Note that $J_{m-M}(0)=\delta_{m M}$.

For the CBB used in Sec. III, the field is defined by the acoustic potential (with time harmonics omitted) as ${ }^{17}$

$$
\Phi_{i}(x, y, z)=\Phi_{0} i^{M} e^{i k_{\|}\left(z-z_{0}\right)} J_{M}\left(k_{\perp} R^{\prime}\right) e^{i M \varphi^{\prime}} .
$$

Note that the relation between acoustic potential and pressure is $p=i \omega \rho_{0} \Phi$, so that there is a coefficient difference $i^{M+1}$ between Eqs. (13) and (C13).

${ }^{1}$ D. Baresch, J.-L. Thomas, and R. Marchiano, "Orbital angular momentum transfer to stably trapped elastic particles in acoustical vortex beams," Phys. Rev. Lett. 121(7), 074301 (2018).

${ }^{2}$ Z. Gong, P. L. Marston, and W. Li, "Reversals of acoustic radiation torque in Bessel beams using theoretical and numerical implementations in three dimensions," Phys. Rev. Appl. 11(6), 064022 (2019).

${ }^{3}$ T. G. Wang, H. Kanber, and I. Rudnick, "First-order torques and solid body spinning velocities in intense sound fields," Phys. Rev. Lett. 73(1), 128-130 (1977).

${ }^{4} \mathrm{~F}$. H. Busse and T. G. Wang, "Torque generated by orthogonal acoustic waves-theory,” J. Acoust. Soc. Am. 69(6), 1634-1638 (1981).

${ }^{5}$ L. Zhang and P. L. Marston, "Acoustic radiation torque on small objects in viscous fluids and connection with viscous dissipation," J. Acoust. Soc. Am. 136(6), 2917-2921 (2014).

${ }^{6} \mathrm{C}$. Eckart, "Vortices and streams caused by sound waves," Phys. Rev. 73(1), 68-76 (1948).

${ }^{7}$ A. Anhäuser, R. Wunenburger, and E. Brasselet, "Acoustical rotational manipulation using orbital angular momentum transfer," Phys. Rev. Lett. 109, 034301 (2012).

${ }^{8}$ A. Riaud, M. Baudoin, J. Thomas, and O. Bou Matar, "Cyclones and attractive streaming generated by acoustical vortices," Phys. Rev. E 90, 013008 (2014).

${ }^{9}$ G. Maidanik, "Torques due to acoustical radiation pressure," J. Acoust. Soc. Am. 30(7), 620-623 (1958).

${ }^{10}$ L. Zhang and P. L. Marston, "Acoustic radiation torque and the conservation of angular momentum (L),” J. Acoust. Soc. Am. 129(4), 1679-1680 (2011).

${ }^{11}$ L. Zhang and P. L. Marston, "Angular momentum flux of nonparaxial acoustic vortex beams and torques on axisymmetric objects," Phys. Rev. E 84(6), 065601 (2011).

${ }^{12}$ B. T. Hefner and P. L. Marston, "An acoustical helicoidal wave transducer with applications for the alignment of ultrasonic and underwater systems," J. Acoust. Soc. Am. 106(6), 3313-3316 (1999).

${ }^{13}$ L. Zhang and P. L. Marston, "Geometrical interpretation of negative radiation forces of acoustical bessel beams on spheres," Phys. Rev. E 84(3), 035601 (2011).

${ }^{14} \mathrm{~L}$. Zhang, "Reversals of orbital angular momentum transfer and radiation torque," Phys. Rev. Appl. 10(3), 034039 (2018).

${ }^{15}$ G. Silva, T. Lobo, and F. Mitri, "Radiation torque produced by an arbitrary acoustic wave," Europhys. Lett. 97(5), 54003 (2012).
${ }^{16}$ D. Baresch, J.-L. Thomas, and R. Marchiano, "Three-dimensional acoustic radiation force on an arbitrarily located elastic sphere," J. Acoust. Soc. Am. 133(1), 25-36 (2013).

${ }^{17}$ Z. Gong, P. L. Marston, W. Li, and Y. Chai, "Multipole expansion of acoustical Bessel beams with arbitrary order and location," J. Acoust. Soc. Am. 141(6), EL574-EL578 (2017).

${ }^{18}$ L. Zhang, "A general theory of arbitrary Bessel beam scattering and interactions with a sphere," J. Acoust. Soc. Am. 143(5), 2796-2800 (2018).

${ }^{19}$ D. Zhao, J.-L. Thomas, and R. Marchiano, "Computation of the radiation force exerted by the acoustic tweezers using pressure field measurements," J. Acoust. Soc. Am. 146(3), 1650-1660 (2019).

${ }^{20} \mathrm{G}$. T. Silva, "Off-axis scattering of an ultrasound Bessel beam by a sphere," IEEE Trans. Ultrason. Ferroelectr. Freq. Control 58(2), 298-304 (2011).

${ }^{21}$ O. A. Sapozhnikov and M. R. Bailey, "Radiation force of an arbitrary acoustic beam on an elastic sphere in a fluid," J. Acoust. Soc. Am. 133(2), 661-676 (2013)

${ }^{22}$ N. Jimenez, R. Pico, V. Sanchez-Morcillo, V. Romero-Garcia, L. Garcia-Raffi, and K. Staliunas, "Formation of high-order acoustic bessel beams by spiral diffraction gratings," Phys. Rev. E 94(5), 053004 (2016).

${ }^{23}$ A. Riaud, M. Baudoin, O. Bou Matar, L. Becerra, and J.-L. Thomas, "Selective manipulation of microscopic particles with precursors swirling Rayleigh waves," Phys. Rev. Appl. 7, 024007 (2017).

${ }^{24}$ N. Jimenez, V. Romero-Garcia, L. Garcia-Raffi, F. Camarena, and K. Staliunas, "Sharp acoustic vortex focusing by Fresnel-spiral-zone plates," Appl. Phys. Lett. 112(20), 204101 (2018).

${ }^{25}$ M. Baudoin, J.-C. Gerbedoen, A. Riaud, O. B. Matar, N. Smagin, and J.L. Thomas, "Folding a focalized acoustical vortex on a flat holographic transducer: Miniaturized selective acoustical tweezers," Sci. Adv. 5(4), eaav1967 (2019).

${ }^{26}$ K. Melde, A. G. Mark, T. Qiu, and P. Fischer, "Holograms for acoustics," Nature 537(7621), 518-522 (2016).

${ }^{27}$ M. Baudoin, J.-L. Thomas, R. Al Sahely, J.-C. Gerbedoen, Z. Gong, A. Sivery, O. B. Matar, N. Smagin, P. Favreau, and A. Vlandas, "Spatially selective manipulation of cells with single-beam acoustical tweezers," Nat. Commun. 11, 4244 (2020).

${ }^{28}$ P. L. Marston, "Scattering of a Bessel beam by a sphere," J. Acoust. Soc. Am. 121(2), 753-758 (2007).

${ }^{29}$ J. J. Faran, Jr., "Sound scattering by solid cylinders and spheres," J. Acoust. Soc. Am. 23(4), 405-418 (1951).

${ }^{30} \mathrm{P}$. Waterman, "New formulation of acoustic scattering," J. Acoust. Soc. Am. 45(6), 1417-1429 (1969).

${ }^{31}$ A. Boström, "Scattering of acoustic waves by a layered elastic obstacle in a fluid-An improved null field approach," J. Acoust. Soc. Am. 76(2), 588-593 (1984).

${ }^{32}$ V. Varadan, A. Lakhtakia, and V. Varadan, "Comments on recent criticism of the t-matrix method," J. Acoust. Soc. Am. 84(6), 2280-2284 (1988).

${ }^{33}$ R. Lim, "A more stable transition matrix for acoustic target scattering by elongated objects," J. Acoust. Soc. Am. 138(4), 2266-2278 (2015).

${ }^{34}$ Z. Gong, W. Li, F. G. Mitri, Y. Chai, and Y. Zhao, "Arbitrary scattering of an acoustical Bessel beam by a rigid spheroid with large aspect-ratio," J. Sound Vibr. 383, 233-247 (2016).

${ }^{35}$ W. Li, Y. Chai, Z. Gong, and P. L. Marston, "Analysis of forward scattering of an acoustical zeroth-order bessel beam from rigid complicated (aspherical) structures," J. Quant. Spectrosc. Radiat. Transf. 200, 146-162 (2017).

${ }^{36}$ M. Baudoin and J.-L. Thomas, "Acoustic tweezers for particle and fluid micromanipulation," Ann. Rev. Fluid Mech. 52, 205-234 (2020).

${ }^{37}$ Z. Gong and M. Baudoin, "Equivalence between angular spectrumbased and multipole expansion-based formulas of the acoustic radiation force and torque," J. Acoust. Soc. Am. (submitted).

${ }^{38} \mathrm{G}$. Gaunaurd and H. Überall, "Theory of resonant scattering from spherical cavities in elastic and viscoelastic media," J. Acoust. Soc. Am. 63(6), 1699-1712 (1978).

${ }^{39}$ B. Hartmann and J. Jarzynski, "Ultrasonic hysteresis absorption in polymers," J. Appl. Phys. 43(11), 4304-4312 (1972). 
${ }^{40}$ Y. Takagi, T. Hosokawa, K. Hoshikawa, H. Kobayashi, and Y. Hiki, "Relaxation of polystyrene near the glass transition temperature studied by acoustic measurements,” J. Phys. Soc. Jpn. 76(2), 024604 (2007).

${ }^{41}$ D. Baresch, J.-L. Thomas, and R. Marchiano, "Spherical vortex beams of high radial degree for enhanced single-beam tweezers," J. Appl. Phys. 113(18), 184901 (2013).

${ }^{42}$ D. Baresch, J.-L. Thomas, and R. Marchiano, "Observation of a singlebeam gradient force acoustical trap for elastic particles: Acoustical tweezers," Phys. Rev. Lett. 116(2), 024301 (2016).

${ }^{43}$ A. Boström, "Multiple scattering of elastic waves by bounded obstacles," J. Acoust. Soc. Am. 67(2), 399-413 (1980).

${ }^{44}$ J. H. Lopes, M. Azarpeyvand, and G. T. Silva, "Acoustic interaction forces and torques acting on suspended spheres in an ideal fluid," IEEE Trans. Ultrason. Ferroelectr. Freq. Control 63(1), 186-197 (2016).
${ }^{45}$ A. Marzo, M. Caleap, and B. W. Drinkwater, "Acoustic virtual vortices with tunable orbital angular momentum for trapping of Mie particles," Phys. Rev. Lett. 120(4), 044301 (2018).

${ }^{46}$ S. Inoue, S. Mogami, T. Ichiyama, A. Noda, Y. Makino, and H. Shinoda, "Acoustical boundary hologram for macroscopic rigid-body levitation," J. Acoust. Soc. Am. 145(1), 328-337 (2019).

${ }^{47} \mathrm{Z}$. Gong, Y. Chai, and W. Li, "Reversals of acoustic radiation force and torque in a single Bessel beam: Acoustic tweezers numerical toolbox," in Proceedings of the Acoustofluidics 2018, Lille, France (August 29-31, 2018), pp. 114-115.

${ }^{48}$ G. B. Arfken, H. J. Weber, and F. E. Harris, Mathematical Methods for Physicists, 7th ed. (Academic Press, New York, 2013), pp. 756-765.

${ }^{49}$ J. D. Jackson, Classical Electrodynamics, 3th ed. (John Wiley \& Sons, New York, 1999), p. 428. 